\title{
Wall Motion Classification of Stress Echocardiography Based on Combined Rest-and-Stress Data
}

\author{
Sarina Mansor, Nicholas P. Hughes, and J. Alison Noble \\ Biomedical Image Analysis (BioMedIA) Laboratory, Institute of Biomedical Engineering, \\ Department of Engineering Science, University of Oxford, United Kingdom \\ \{sarina, nph, noble\} @robots.ox.ac.uk \\ http: //www.ibme.ox.ac.uk/biomedia
}

\begin{abstract}
In this paper, we represent a new framework that performs automated local wall motion analysis based on the combined information derived from a rest and stress sequence (a full stress echocardiography study). Since cardiac data inherits time-varying and sequential properties, we introduce a Hidden Markov Model (HMM) approach to classify stress echocardiography. A wall segment model is developed for a normal and an abnormal heart and experiments are performed on rest, stress and rest-and-stress sequences. In an assessment using $n=44$ datasets, combined rest-and-stress analysis shows an improvement in classification $(84.17 \%)$ over individual rest $(73.33 \%)$ and stress $(68.33 \%)$.
\end{abstract}

\section{Introduction}

Automated quantitative wall motion analysis aims to further improve accuracy, decrease subjectivity and increase reproducibility of interpretation of echocardiography over visual interpretation. While much has been done to attempt to automate the task of wall motion analysis on rest image sequences, there is a very small literature on analysis of stress echocardiography. Stress sequences (where the heart has been stressed by exercise or use of a pharmacological drug e.g. dobutamine) are more difficult to automatically analyse as the non-rigid motion of the heart is more challenging to track.

The first step of automated regional heart function analysis is to detect the heart wall borders. Many techniques have been developed to detect and automatically track both the interior (endocardial) and exterior (epicardial) borders of the left ventricle (LV). Current automated 2D echocardiography image tracking technology is now sufficiently well-developed for application on good-to-medium quality rest data [1]. However, further studies need to be done to show that this is also true for stress echocardiography. In the work reported here, we used semi-automated derived contours as our initial focus has been on classification not automated tracking.

The second step of automated regional heart function analysis is classification of heart segmental function as either normal or abnormal based on the extracted contours (heart wall borders). This is the focus of this paper. We have proposed a new approach to wall motion classification of stress echocardiography based on Hidden Markov Models (HMMs) in our previous works [2,3] and good results were obtained. The main reason we chose HMMs over other classification methods is due to time-varying and 
sequential properties of cardiac data. HMMs allow us to capture the temporal relations and ordering between cardiac sequences. There are other works in classifying heart wall motion using shape models [4] and Bayesian network [5] - but they only consider rest sequences only (i.e.: images taken when a patient is resting).

In our recent work [3], we performed local wall motion classification on individual rest and stress sequences separately. However, in clinical practice, cardiologists make a visual side-by-side comparison of rest and stress images [6]. Inspired by this, we extend the analysis to classify heart motion based on combined rest and stress sequence, which is the main contribution of this paper. To our knowledge, there has been no prior attempt to do heart disease classification based on the combined information of rest and stress in the literature. We are only aware of work looking individually at either rest or alternatively stress data but not the combination of both [7].

\section{Hidden Markov Models}

A detailed tutorial on HMMs can be found in [8] and a brief description based on that paper is given here. A hidden Markov model (HMM) is a probabilistic model which describes the statistical relationship between an observable sequence $\mathrm{O}$ and a "hidden" state sequence $\mathrm{S}$. The hidden state is discrete, but the observation values may be either continuous or discrete in nature. An HMM is characterized by the following parameters:

1. The number of states of the model, $\mathrm{N}$.

2. The state transition matrix, A

3. The observation probability distribution, $B=\left\{b_{N}\right\}$ for each state $N$

4. The initial state distribution, $\pi$

There are three basic problems associated with HMMs [8]: 1) the classification/evaluation problem, 2) the decoding problem and 3) the learning/training problem. In our work we deal with the first and third problems. A regional heart function model needs to be learned from a training set by the EM algorithm before using the forward-backward procedure for classification of a new dataset.

\section{Methodology}

\subsection{Patient Data}

The available database consisted of 44 studies of Contrast DSE (Dobutamine Stress Echocardiography) data acquired by two cardiologists as standard B-mode ultrasound image sequences. In this paper, we utilized the 2-chamber $(2 \mathrm{C})$ view data at the resting phase and at the peak level of stress. In a standard stress examination, only the systolic (heart contracts) phase is recorded. Therefore, each data consists of systole only starting from the end-of-diastolic (ED) phase to the end-of-systolic (ES) phase.

For regional functional analysis, the $2 \mathrm{C}$ is divided into 7 segments but mostly only 6 are measurable (especially for abnormal hearts) [9]. Clinical wall functional assessment evaluates systolic thickening $^{1}$ and endocardial wall motion for each segment. A normal (healthy) myocardial segment shows systolic thickening and also endocardial movement

\footnotetext{
${ }^{1}$ The myocardial wall thickening during systole (the contraction of the heart) phase.
} 
towards the centre of the cavity. Each of 6 segments were labelled 1 - $4(1=$ normal, $2=$ hypokinesis, 3 = akinetic, 4 = dyskinetic). For simplicity, we grouped them into 2 classes only ( 1 = normal, $2-4$ = abnormal $)$.

\subsection{Wall Motion Classification}

Fig. 1 shows the general process of our HMM-based wall motion classification method which can be summarised as follows:

1. Firstly, the myocardial contours were extracted by Quamus ${ }^{2}{ }^{2}$ software. These were then validated by two experienced cardiologists.

2. Each contour was then divided into six segments (for $2 \mathrm{C}$ data) and a number of features estimated to characterize cardiac wall motion (e.g.: wall thickening, cavity area, endocardial motion).

3. The cardiac data was separated into two groups based on wall motion scoring: Normal (score =1), Abnormal (score = 2-4).

4. Two HMMs were then developed for each segment: one for normals and one for abnormals. Thus, there were 12 trained models in total.

5. Finally, the trained HMMs (models) were used to classify a new (unseen) dataset.

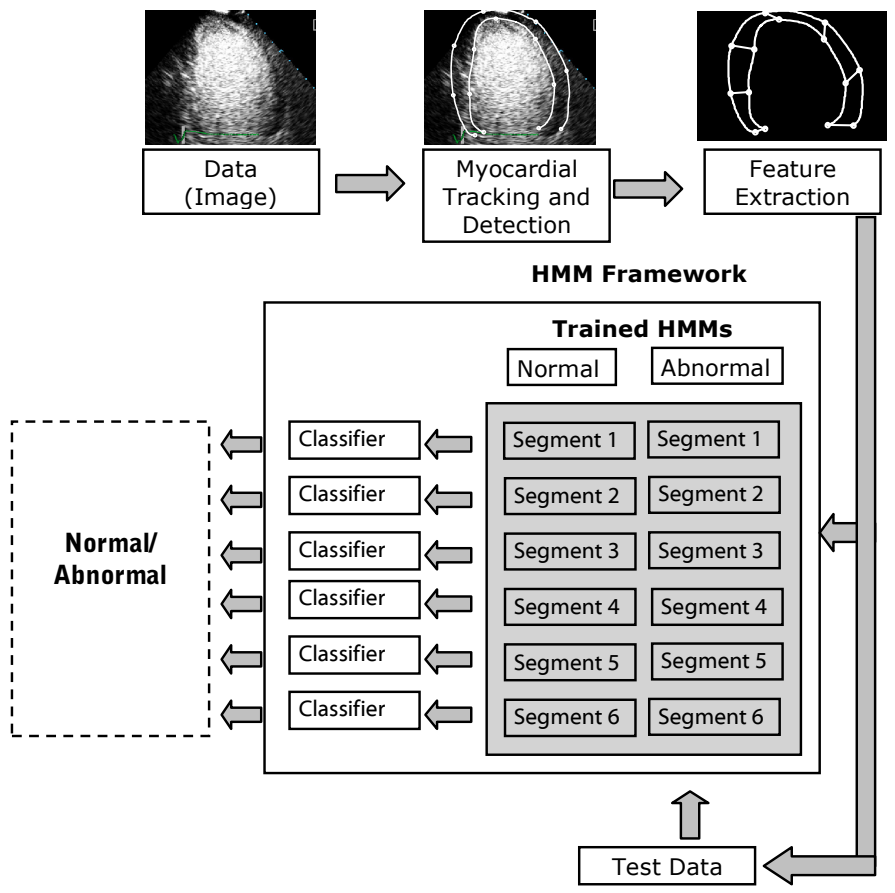

Fig. 1. Diagram of Wall Motion Classification Process

${ }^{2}$ A semi-automatic boundary detection software developed by Mirada Solutions Ltd, Oxford, United Kingdom. 


\subsection{Feature Vector / Object Representation}

As mentioned earlier, several features could be used to detect segmental wall motion abnormalities. In this paper, we chose the 'local cavity area' (area under endocardial contour) for each of six segments, as shown in Fig. 2.

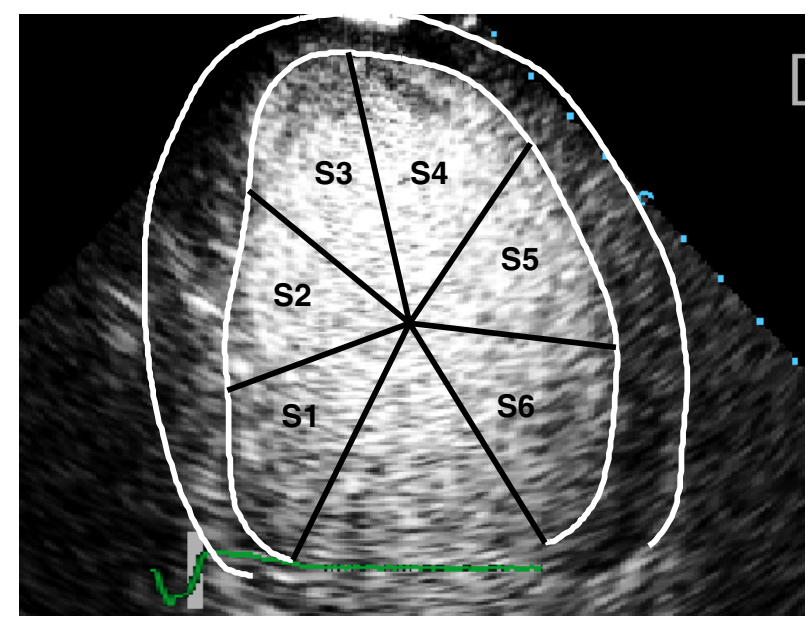

Fig. 2. Local Cavity Area for all six segments of $2 \mathrm{C}$ data ( $i=$ local cavity area for Segment $i$, where $i=1,2, . ., 6$ )

This feature vector was suggested by our cardiologist collaborators as it is the simplest and easiest observation representation that can be viewed from all echocardiographic data. Furthermore, we need only endocardial contours (which are easier to track/segment than epicardial contours) and centre of cavity in order to compute the local cavity area. The local cavity area (LCA) is then normalised by the following equation (1):

$$
L C A(i)=\frac{L C A_{E D}-L C A(i)}{L C A_{E D}}
$$

where $L C A_{E D}=$ Local Cavity Area at End of Diastolic.

For individual rest or stress sequence analysis (cases 1 and 2 respectively in our study), we compose a feature/observation as a $1 \mathrm{D}$ vector, as follows:

$$
O_{R E S T \text { (or STRESS) }}=[\underbrace{O_{f 1}, O_{f 2} \cdots, O_{f N}}_{\text {Patient } 1}, \underbrace{O_{f 1}, O_{f 2}, \cdots, O_{f N}}_{\text {Patient } 2}, \cdots \cdots \underbrace{O_{f 1}, O_{f 2} \cdots, O_{f N}}_{\text {Patient } K}]^{\mathrm{T}}
$$

where $O_{f i}$ is the observation at frame $i$, which in our experiment is equivalent to $L C A(i)$. 
In the case of combined 'rest-and-stress' sequences (case 3), we combined together $O_{R E S T}$ and $O_{\text {STRESS }}$ as a $2 \mathrm{D}$ vector:

$$
O_{\text {REST-STRESS }}=\left[O_{\text {REST }} \mid O_{\text {STRESS }}\right]
$$

The main reason we modelled this as a $2 \mathrm{D}$ vector (rather than a concatenate $1 \mathrm{D}$ vector) is to preserve the temporal dependency between $O_{R E S T}$ and $O_{S T R E S S}$ (as our results show later this turns out to be important).

\subsection{Training}

The sequential nature of the heart phases can be well modelled by a left-right HMM, which has been widely used for speech recognition [8]. We developed two models for each segment: one for normals and another for abnormals. Each model is comprised of three hidden states. In our case, the states of the HMM do not have a true physical meaning - they simply reflect common statistical properties of the observation vectors in the feature space. We could have employed more states but that would require more training samples for an accurate estimation of the model parameters [10].

We trained the two HMMs (one for normals, one for abnormals) in an unsupervised manner. Prior to training, all datasets were resampled to have 30 frames per patient. We then calculated the normalised local cavity area for each frame, as previously described in Section 3.3.

In the first stage of training, we initialised the model parameters as follows. For each patient in the dataset, the first ten frames were assigned to state 1 , the next ten to state 2 and the final ten to state 3 . The parameters of the transition matrix $a_{i j}$ were then initialised using the maximum likelihood estimates, based on the following equation:

$$
a_{i j}=\frac{n_{i j}}{\sum_{k} n_{i k}}
$$

where $n_{i j}$ is the total number of transitions from state $i$ to state $j$ over all of the label sequences.

The observation probability densities $b_{i}$ (which were Gaussian) were initialised by fitting either 1D or 2D Gaussian to the set of features extracted from the frames assigned to each state $i$. Following initialisation of the HMM parameters, we then ran the EM algorithm to update the model parameters until the change in the log likelihood was less than $0.01 \%$ (indicating convergence).

For classification purposes, we compute the posterior probability, p(modelldata) for all models and the maximum value gives the most probable model for the data. The posterior probability can be computed by using Bayes' rule:

$$
p(\text { model } \mid \text { data })=\frac{p(\text { data } \mid \text { model }) p(\text { model })}{\sum_{\text {model }} p(\text { data }, \text { model })}
$$

where $p$ (datalmodel) is computed using the forward-backward algorithm and $p($ model $)$ is the prior probability for each model. 


\section{Results and Discussion}

The HMM-based approach was evaluated using two classification experiments: 1) a leave-one-out cross validation and 2) a new dataset testing experiment. Recall that we have a total of 44 studies (patients), with normal and abnormal cases. We divided them into training and testing dataset. For training purpose, we chose patients with severe abnormality (wall motion score is above one for all segments) in order to produce a good Abnormal trained model. Therefore, we have 12 Normals and 12 Abnormals for training set and the remaining 20 (which is a mixed of normal and mild abnormality cases) are used for testing set.

In a leave-one-out cross-validation one of the 12 sequences for the model being tested is held out and the remaining 11 sequences are used to derive the HMM. The held-out sequence is then tested on this HMM and the other fully trained HMM. Table 1 shows the classification accuracy results for rest, stress and 'combined rest-stress' data using both global and local classification approach. The global classification method can be found in our previous paper [2].

In the testing experiment, we computed the posterior probability that each model (Normal and Abnormal) gives to the test sequences and classified them based on equation (5) (refer to Section 3.4). Table 2 gives the classification accuracy of the 20 testing sets for both rest and stress data.

Table 1. Percentage global and local classification accuracy of rest, stress and combined restand-stress sequences using a leave-one-out approach

\begin{tabular}{cccccccc}
\hline & & \multicolumn{2}{c}{$\begin{array}{c}\text { REST } \\
\text { Classification (\%) }\end{array}$} & \multicolumn{2}{c}{$\begin{array}{c}\text { STRESS } \\
\text { Classification (\%) }\end{array}$} & \multicolumn{2}{c}{$\begin{array}{c}\text { REST-STRESS } \\
\text { Classification (\%) }\end{array}$} \\
\hline & & N & A & N & A & N & A \\
\hline GLOBAL & ALL & 100 & 83.3 & 83.3 & 91.7 & 100 & 75 \\
\hline \multirow{5}{*}{ LOCAL } & S1 & 91.7 & 100 & 91.7 & 91.7 & 91.7 & 91.7 \\
& S2 & 100 & 100 & 83.3 & 91.7 & 91.7 & 83.3 \\
& S3 & 83.3 & 91.7 & 83.3 & 91.7 & 83.3 & 83.3 \\
& S4 & 91.7 & 83.3 & 83.3 & 91.7 & 100 & 75 \\
& S5 & 100 & 83.3 & 75 & 91.7 & 100 & 83.3 \\
& S6 & 100 & 83.3 & 75 & 91.7 & 100 & 83.3 \\
\hline
\end{tabular}

The purpose of using a leave-one-out approach is to evaluate the classification performance and also useful in finding a good feature observation for quantitative wall motion analysis. As can be seen from the Table 1, we can achieve more or less the same accuracy of classification for the rest-and-stress sequences as well as the individual rest ones and an improved accuracy over stress data.

As for the testing experiment (refer to Table 2), a good accuracy of classification was achieved even with this small number of datasets in the trained models. This shows the potential use of HMM for classification. The more interesting result lies on the analysis of rest-to-stress sequence where it had higher average accuracy $(84.17 \%$ and $79.17 \%$ when the combined data is modelled as 1D and 2D vectors respectively) than that of the individual rest $(73.33 \%)$ and stress $(68.33 \%)$ sequences. This is 
Table 2. Percentage classification accuracy of rest, stress and combined rest-and-stress sequences on testing dataset

\begin{tabular}{ccccc}
\hline SEGMENT & REST $(\%)$ & STRESS $(\%)$ & \multicolumn{2}{c}{ REST-STRESS (\%) } \\
& & & 80 & 85.0 \\
(1D Vector) & (2D Vector) \\
\hline S1 & 65.0 & 75.0 & 95 & 90.0 \\
S2 & 80.0 & 85.0 & 80 & 80.0 \\
S3 & 70.0 & 70.0 & 60 & 65.0 \\
S4 & 60.0 & 60.0 & 85 & 100.0 \\
S5 & 80.0 & 70.0 & 75 & 85.0 \\
S6 & 85.0 & 50.0 & $\mathbf{7 9 . 1 7}$ & $\mathbf{8 4 . 1 7}$ \\
\hline Mean & $\mathbf{7 3 . 3 3}$ & $\mathbf{6 8 . 3 3}$ &
\end{tabular}

encouraging evidence to pursue the combined analysis of rest-and-stress data. Table 2 also indicates that modelling the combined rest-and-stress data in $2 \mathrm{D}$ vector is better than in a concatenate 1D vector, as the temporal dependency can be preserved (as discussed in Section 3.3).

\section{Conclusions}

In this paper, we have proposed a framework for heart disease classification based on combined rest and stress sequences. We utilized the new approach of local wall motion classification based on HMMs. An $11 \%$ improvement of accuracy has been achieved when we performed classification on the combined rest-and-stress data compared to the separate individual sequences. These results are consistent with cardiologists' experience, where they read rest and peak stress data together in clinical decision-making. These encouraging results could be improved further by: 1) increasing the number of training data, for example at least 50 patients for each model, 2) developing different models for different types of abnormality and 3) combining with 'mid-level peak' and 'recovery' sequences, which usually been recorded as well during stress testing. The general approach would be equally applicable to 3D stress echocardiography and the methodology is not modality specific.

Acknowledgements. Financial support from Telekom Malaysia is acknowledged. We thank Dr H Becher and Dr Szmigielski (Oxford JR Hospital) for providing patient data, data analysis support and useful discussions.

\section{References}

[1] Noble, J.A.: Cardiology meets Image Analysis: just another application or can image analysis usefully influence clinical practice? In: IEEE International Workshop on Computer Vision in Biomedical Image Analysis, Beijing, China (2005)

[2] Mansor, S., Noble, J.A., Hughes, N.P.: Regional Left Ventricular Wall Motion Analysis using Hidden Markov Models. In: Proceedings of Medical Image Understanding and Analysis, Wales, United Kingdom, pp. 136-140 (2007) 
[3] Mansor, S., Noble, J.A.: Local Wall Motion Classification of Stress Echocardiography using Hidden Markov Models. In: IEEE International Symposium on Biomedical Imaging, ISBI 2008, Paris, France (2008)

[4] Esther Leung, K.Y., Bosch, J.G.: Local Wall Motion Classification in Echocardiograms using Shape Models and Orthomax Rotations. In: Sachse, F.B., Seemann, G. (eds.) FIHM 2007. LNCS, vol. 4466, pp. 1-11. Springer, Heidelberg (2007)

[5] Qazi, M., Fung, G., et al.: Automated Heart Wall Motion Abnormality Detection from Ultrasound Images Using Bayesian Networks. In: International Joint Conferences on Artificial Intelligence, IJCAI 2007, Hyderabad, India (2007)

[6] Picano, E.: Stress Echocardiography, $4^{\text {th }}$ edn. Springer, Heidelberg (2003)

[7] Suinesiaputra, A., Frangi, A.F., et al.: Automatic Prediction of Myocardial Contractility Improvement in Stress MRI Using Shape Morphometrics with Independent Component Analysis. In: Christensen, G.E., Sonka, M. (eds.) ICATPN 2005. LNCS, vol. 3536, pp. 321-332. Springer, Heidelberg (2005)

[8] Rabiner, L.: A Tutorial on Hidden Markov Models and Selected Applications in Speech Recognition. Proceedings of the IEEE 77(2), 257-286 (1989)

[9] Lang, R.M., et al.: Recommendations for chamber quantification. European Journal Echocardiography 7, 79-108 (2006)

[10] He, Y., Kundu, A.: 2-D Shape Classification Using Hidden Markov Model. IEEE Trans. IEEE Trans. Pattern Analysis and Machine Intelligence 13(1), 1172-1184 (1991) 